\title{
Study on a Supervisory System for Military Hardware Operation in Low Light Conditions \\ Jun Yang ${ }^{1}$, Shicong $\operatorname{Lin}^{1, a}$ \\ ${ }^{1 .}$ Academy of Armored Force Engineering, Beijing 100072, China \\ a.346247941@qq.com
}

Keywords: low light conditions, hardware operation, image de-noising, image enhancement

\begin{abstract}
Aimed at the issue of the operation training on military hardware, designed a set of supervisory system for military hardware operation in low light conditions. Put forward an image denoising algorithm which united mathematics morphology and modified average filtering method, put forward an image enhancement algorithm which united histogram equalization method and modified local contrast enhancement method, used wireless communication technology to realize remote realtime monitoring. By experiment, the two algorithms can solve the problem of low illumination image's poor quality, the system can realize the real-time communication between the command and control center and the fighting vehicles, the real-time monitoring of the image inside the vehicles, and other functions, meaningfully to complete the operation training on military hardware in low light conditions, and provided the theoretical basis and technical reference for other training monitoring systems.
\end{abstract}

\section{Introduction}

With the progress of science and technology, the technology intensity and operation complexity of military hardware is higher and higher. Some weapons bring big trouble to operation training because of the requirements of overall dimensions, the internal space reserved for operating personnel is little and the brightness is not enough when close the hatch. Aiming at these problems, we design a set of supervisory system for military hardware operation in low light conditions, which can give the training course to trainees, take the images of trainees, the real-time dialogue between organization and trainees, and other important features. It has great significance for operation training on military hardware.

The technical key point of this system is the design of the algorithm for image acquisition and handling method under low illumination environment. On the basis of improve the system, the article put forward an image de-noising algorithm which united mathematics morphology and modified average filtering method, put forward an image enhancement algorithm which united histogram equalization method and modified local contrast enhancement method, to solve the problem of low illumination image.

\section{OVERALL DESIGN OF SYSTEM}

Supervisory system for military hardware operation dominated by LINUX embedded video monitoring system based on the WLAN, generally divided into three parts: military hardware 、 repeater、command and control center(see Figure 1). Hardware devices is composed of cameras、 media processing platform、microphones and speakers、wireless network card and AP contact、 PC and storage device、 cable, etc.

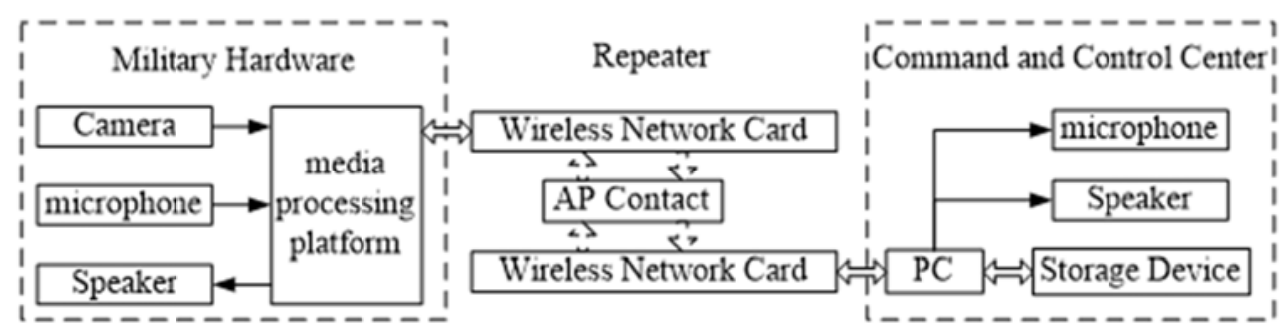

Fig.1 System structural framework 
The technical key point of the system is the image acquisition and handling method under low illumination environment. Aimed at the problem of low illumination image such as lower resolution, large noise and difficult edge feature extraction. We choose a 1/2 inch CDD high resolution camera to acquire image, make sure the high resolution; put forward an image de-noising algorithm and an image enhancement algorithm to process low illumination images.

\section{DESIGN OF IMAGE DE-NOISING ALGORITHM}

Mathematical morphology analysis the image based on the morphological structuring elements, it measures and extracts the shape of image by structural elements with some form. Using this way to denoise can gather the information of image, avoid losing it, but we can't get the motorized model. Average filtering method is a kind of linear filtering algorithm, de-noising effect is good, but it damages the detail of image, makes the image blur.

Comprehensive the above two methods, the system designs the algorithm as follows: first use mathematical morphology filter out part of noise and gather the information; then use the modified average filtering method filter out noise deeply, at the moment the information of image can be protected well.

The modified average filtering method choose a $3 \times 3$ two dimensional slide template (see Figure 2 ) to process image, will a grey value of pixels in the original image sum up with the surrounding eight grayscale values of the pixels and weighted after averaging, retrieves the pixel gray value in the new image. After dispose a certain point $(x, y)$ has a new pixel value $h(x, y) . h(x, y)$ can be calculated by type (1).

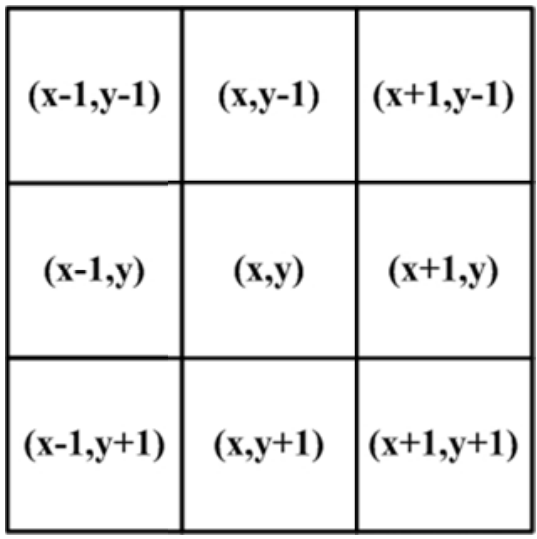

Fig. 2 Template $3 \times 3$

$$
\mathrm{h}(\mathrm{x}, \mathrm{y})=\frac{\left\{\begin{array}{c}
f(x-1, y-1)+f(x, y-1)+f(x+1, y-1)+f(x-1, y) \\
+f(x, y)+f(x+1, y)+f(x-1, y+1)+f(x, y+1)+f(x+1, y+1)
\end{array}\right\}}{9}+\mathrm{k}
$$

Some code of modified average filtering method as follows:

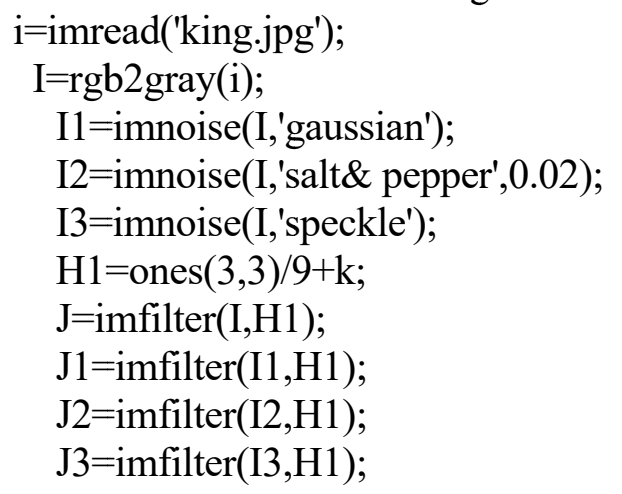




\section{DESIGN OF IMAGE ENHANCEMENT ALGORITHM}

The commonly used image enhancement methods are histogram equalization method and the local contrast image enhancement method. In the experiment, when using the histogram equalization method process image, the enhancement effect is difficult to control, the details of image usually is sacrificed by the global; when using the local contrast image enhancement method, the enhancement effect of uneven low contrast image don't ideal, amplification coefficient in the enhancement process do not adjustable.

In conclusion, Combine with the actually characteristics of the system, we improve the local contrast image enhancement method, and put forward an image enhancement algorithm which united histogram equalization method and modified local contrast enhancement method, the former of the algorithm improves image appearance the latter enhance image details. The local contrast image enhancement method is expressed by type (2).

$$
\mathrm{h}(\mathrm{x}, \mathrm{y})=\left(\mathrm{g}(\mathrm{x}, \mathrm{y})-\mathrm{M}_{\mathrm{x}, \mathrm{y}}\right) \times \mathrm{k}+\mathrm{M}_{\mathrm{x}, \mathrm{y}}
$$

Where, $\mathrm{k}$ is the amplification coefficient; $\mathrm{g}(\mathrm{x}, \mathrm{y})$ is the mapping function of grey level for point $(x, y)$ after the histogram equalization method processing; $h(x, y)$ is the grey level for point $(x, y)$ after the local contrast image enhancement; $M_{x, y}$ is the average grey level centering on the pixel processing window.

Improvement of type (2), get the type (3).

$$
h(x, y)=M_{\alpha} Z_{M}+\left(1-M_{\alpha}\right) \times g(x, y)+\left(f(x, y)-M_{x, y}\right) \times k^{\prime}
$$

Where, $M_{\alpha}$ is the average adjustment coefficient; $Z_{M}$ is the average expectation of image after enhancement; $\mathrm{k}^{\prime}=0.9+\frac{\operatorname{INT}(\sqrt{255 / \mathrm{g}(\mathrm{x}, \mathrm{y})}}{16}$ is the amplification coefficient after improvement, when $\mathrm{g}(\mathrm{x}, \mathrm{y})=0, \mathrm{k}^{\prime}=1.9$.

The realization method of image enhancement algorithm is as follows:

1. Assume that the grey level of point $(x, y)$ is $f(x, y)$, after the histogram equalization method processing the grey level of point $(x, y)$ is $g(x, y)$;

2. The domain value judgment by type (4).

$$
h(x, y)=\left\{\begin{array}{c}
M_{\alpha} Z_{M}+\left(1-M_{\alpha}\right) \times g(x, y)+\left(f(x, y)-M_{x, y}\right) \times k^{\prime}, \quad 0 \leq f(x, y) \leq 255 \\
M_{\alpha} Z_{M}+\left(1-M_{\alpha}\right) \times g(x, y), \text { others }
\end{array}\right.
$$

where, $M_{\alpha} Z_{M}+\left(1-M_{\alpha}\right) \times g(x, y)$ play the role of the gray-level histogram adjusting dynamic range, $\left(f(x, y)-M_{x, y}\right) \times k^{\prime}$ play the role of the local contrast enhancement refining.

Under different low illumination environment image processing effect test of this system (see Figure 3).

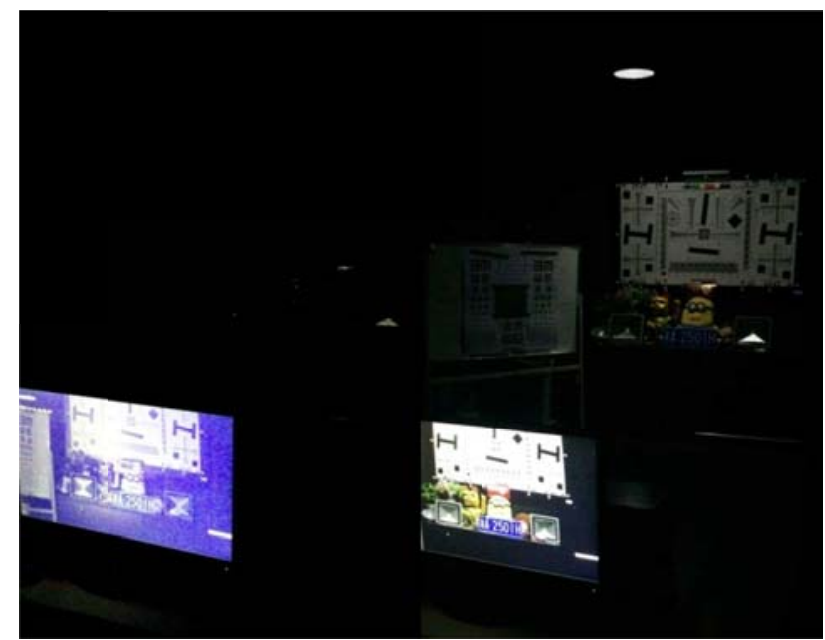

(a) (b)

Fig.3 The effect of image processing 


\section{CONCLUSIONS}

A supervisory system for military hardware operation in low light conditions is designed and implemented in the paper. The specific work and innovation are as follows:

1. Basically completed system overall architecture and design and selection of specific equipment, expectations can be stable and efficient to achieve;

2. Put forward an image de-noising algorithm which united mathematics morphology and modified average filtering method, the algorithm's de-noising effect is good for low illumination image, and will not lost image information in the process of de-noising.

3. Put forward an image enhancement algorithm which united histogram equalization method and modified local contrast enhancement method, the algorithm can achieve good effect.

In conclusion, the system can realize the function of real-time monitoring under low illumination image, meaningfully to complete the operation training on military hardware in low light conditions, and provided the theoretical basis and technical reference for other training monitoring systems.

\section{References}

[1]. Yi San-li, He Jian-feng, Shao Dang-guo, etal. DWI Denoising Method Based on BEMD and Adaptive Wiener Filter[J]. Journal of Data Acquisition and Processing, 2014,29(1):90-94.

[2]. Wang Shu-wei, Yan Cheng-xin, Zhang Tian-xu, etal. Application of Mathenatical Morphology in Image Processing[J]. Computer Engineering and Applications. 2004,32:89-92.

[3]. Sun Sheng, Liu Ren-feng, Wen Wen, etal. Application of Tensor-diffusion Based Filter in Despeckle of Polarimetric Synthetic Aperture Radar Imagery[J]. Science Technology and Engineering. 2015,15(18):190-194.

[4]. Peng Bo, Wang Yi-ming. Research and Implementation of Enhancement Algorithm for Lowillum Ination Image[J]. Computer Applications. 2007,27(8):2001-2003.

[5]. Li Ling-jie, Tang Ning. A Fast Enhancemeng Algorithm for Traffic Sence[J]. Computer and Modernization. 2015,6:55-58.

[6]. Wang Shu-min, Zhang Ai-wu, Hu Shao-xing, etal. The Retinex Algorithm of Image Enhancement Based on the Global and Local Illumination Estimation[J]. Science Technology and Engineering. 2015,15(34):100-104. 